\title{
SGP Cloud and Land Surface Interaction Campaign (CLASIC):
}

\section{Science and Implementation Plan}

June 2007

Mark A. Miller, Principal Investigator

and

The CLASIC Steering Committee:

Roni Avissar, Larry Berg, Sylvia Edgerton, Marc Fischer, Tom Jackson, Bill Kustas, Pete Lamb, Greg McFarquhar, Qilong Min, Beat Schmid, Margaret Torn, and Dave Turner

Work supported by the U.S. Department of Energy, Office of Science, Office of Biological and Environmental Research 


\section{Executive Summary}

The Cloud and Land Surface Interaction Campaign is a field experiment designed to collect a comprehensive data set that can be used to quantify the interactions that occur between the atmosphere, biosphere, land surface, and subsurface. A particular focus will be on how these interactions modulate the abundance and characteristics of small and medium size cumuliform clouds that are generated by local convection. These interactions are not well understood and are responsible for large uncertainties in global climate models, which are used to forecast future climate states. The campaign will be conducted from June 8 to June 30, 2007, at the U.S. Department of Energy's Atmospheric Radiation Measurement Climate Research Facility Southern Great Plains site. Data will be collected using eight aircraft equipped with a variety of specialized sensors, four specially instrumented surface sites, and two prototype surface radar systems. The architecture of Cloud and Land Surface Interaction Campaign includes a highaltitude surveillance aircraft and enhanced vertical thermodynamic and wind profile measurements that will characterize the synoptic scale structure of the clouds and the land surface within the Atmospheric Radiation Measurement Climate Research Facility Southern Great Plains site. Mesoscale and microscale structures will be sampled with a variety of aircraft, surface, and radar observations. 


\section{Contents}

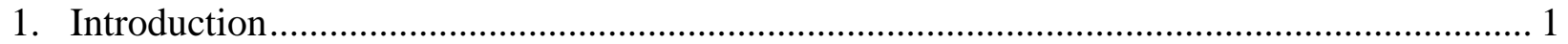

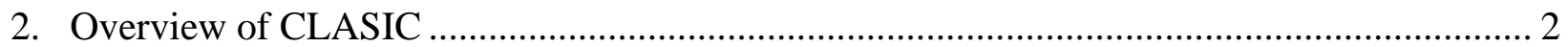

3. CLASIC Science Questions ............................................................................. 4

3.1 Science Question 1-What are the roles of cumulus convection and spatial

variations in land cover in depleting low-level water vapor as it is advected into

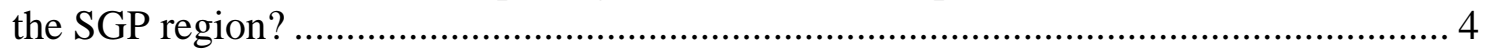

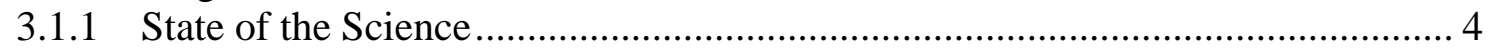

3.1.2 CLASIC Measurement Strategy ............................................................ 6

3.2 Science Question 2-What are the relationships between cumulus clouds and the soil-plant-atmosphere exchange of heat, carbon, and water at the site?....................... 6

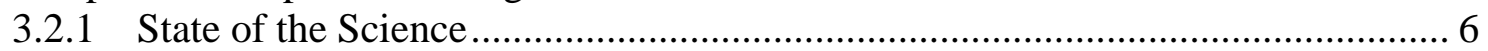

3.2.2 CLASIC Measurement Strategy …............................................................. 7

3.3 Science Question 3-How do land cover changes, such as agricultural harvesting, impact surface heat, carbon, and water fluxes, and can those changes affect local

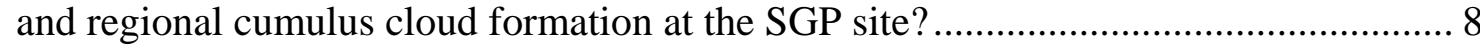

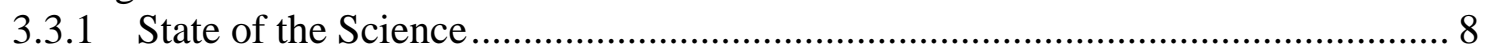

3.3.2 CLASIC Measurement Strategy ................................................................ 8

3.4 Science Question 4-How do land surface processes at the SGP affect atmospheric aerosol loading and chemistry and what are the resulting effects on the microphysical and macrophysical properties of cumulus cloud fields? ............................... 8

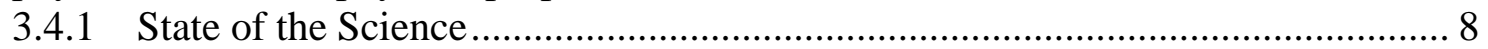

3.4.2 CLASIC Measurement Strategy .............................................................. 9

3.5 Science Question 5-How well do the long-term (+15 years) surface flux measurements made at specific locations within the ACRF SGP represent the actual distribution of the fluxes across the domain? ................................................ 11

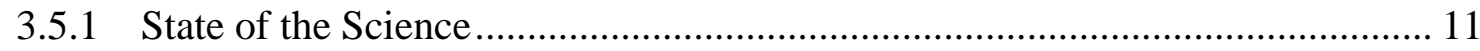

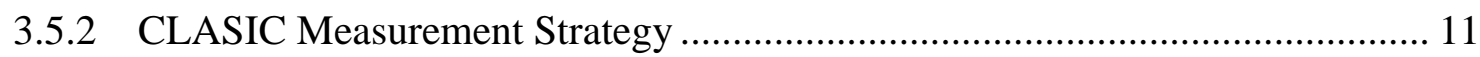

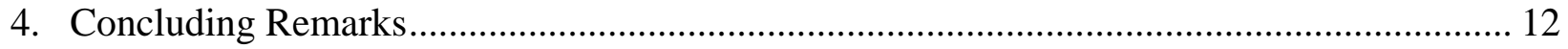

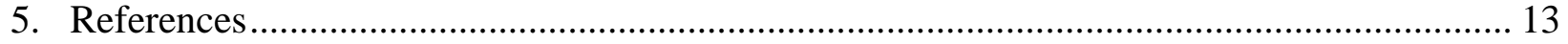




\section{Introduction}

The Intergovernmental Panel for Climate Change has identified land surface forcing to be among the most unknown of all climate-forcing mechanisms through the industrial era since about 1800 . The configuration of the land surface and land use affects exchanges of heat, momentum, water, and carbon between the land surface and atmosphere. Many modeling studies have been conducted to quantify the role of the land surface in the climate system by testing the sensitivity of the climate to changes in a particular land surface property, most notably changes in albedo, soil moisture, surface roughness, and vegetation type. Other modeling studies have related land surface use to the hydrologic cycle and have shown that significant land use changes in a particular area may impact the climate of distant regions through changes in the large-scale circulation that result from altered patterns of atmospheric heating. Modeling and observational studies also have shown that the configuration of the land surface may significantly impact the cloud structure and that the cloud structure may, in turn, impact the configuration of the land surface by altering the radiation budget and precipitation. Cloud and land surface interactions are a particularly vexing issue because of the complexity and variety of land surface, biosphere, and atmospheric processes and exchanges that are involved.

A cloud type that shows particular sensitivity to the underlying land surface is locally generated cumulus convection. Cumulus clouds are estimated to cover approximately 5\% of the global land surface at any given time, which suggests they are important components of the global water and energy budget. These clouds play a key role in the hydrologic cycle, particularly in semi-arid regions and in regions with significant agricultural activity, by regulating the surface radiation budget and providing rainfall during latter stages of their development. Modeling studies show that the timing and evolution of locally generated continental cumulus convection is determined, in part, by the partitioning of the available energy in the atmospheric boundary layer. The available energy may be partitioned such that either the latent heat flux or the sensible heat flux is dominant depending on land surface characteristics such as the soil moisture, vegetation status, albedo, evapotranspiration, aerosol distributions, and land use patterns. The nature of this partition determines characteristics ranging from the time of day when cumulus clouds first form over an area to the structural characteristics of the cloud field that evolves over several days. Simultaneously, the land surface itself is influenced by the cumulus clouds through rain-induced changes in soil moisture and through the impacts of the cumulus cloud field on photosynthesis and, ultimately, evapotranspiration. Thus, locally generated cumulus convection is intimately linked to the land surface coverage through the latent, sensible, and radiative fluxes, which interact through complex pathways that are not yet well understood.

The potential consequences of increasing atmospheric greenhouse gas concentrations for the Earth's climate system usually are evaluated using global climate models (GCMs), which must accurately simulate the complex array of mechanisms and feedbacks in the climate system and predict how they will operate in the future. Most GCMs are unable to accurately simulate cumulus convection, particularly in its early and middle stages. These stages are a particular challenge because the processes that control the development and evolution of the clouds occur at many different scales ranging from microscopic atmospheric condensation rates and plant physiological responses, to patterns of atmospheric updraft that can occur over many kilometers. The role of human activities such as land use in the initiation and maintenance of the early stages 
of convection are only vaguely understood. This knowledge gap is the motivation for the Cloud and Land Surface Interaction Campaign (CLASIC), which is a field program to be held in the Southern Great Plains (SGP) of the United States during June 2007. Cumulus convection is an especially important component of the atmospheric radiation budget and hydrologic cycle in this region, which also is home to the Atmospheric Radiation Measurement (ARM) Program's Climate Research Facility (ACRF) SGP site ${ }^{1}$. The expected result of CLASIC is improved understanding of the physical links between the land surface and cloud structure that can be parameterized and introduced into GCMs to reduce the documented uncertainties currently associated with land surface forcing during the industrial period since about 1800 .

\section{Overview of CLASIC}

The primary goal of CLASIC is to improve understanding of the physics of the early stages of cumulus cloud convection as it relates to land surface conditions, and to translate this new understanding into improved representations in GCMs and regional climate models. The campaign will conduct a comprehensive array of measurements from a variety of instrument platforms, use the resulting data to characterize the synoptic scale forcing at the ACRF SGP site, and undertake modeling studies to establish the most important relationships between land surface conditions and cumulus cloud characteristics.

A three-pronged measurement strategy will be used during CLASIC. First, there will be a large aircraft and surface radar campaign to characterize local variations in the land surface, atmospheric flux profiles, and cloud properties. An ER-2 airplane will fly at altitudes approaching 65,000 ft and serve as the backbone of the campaign. It will measure cloud and precipitation structures while simultaneously measuring land surface properties such as the spectral surface albedo and soil moisture, thereby producing a unique data set that links the land surface and cloud structure on a regional scale. The ER-2 will make frequent surveys of the ACRF SGP site beginning in late-morning and concluding in late afternoon and will be equipped with downward looking cloud $(95-\mathrm{GHz})$ and precipitation $(3-\mathrm{cm})$ radars, a downward looking lidar, and a 50-channel imager known as the moderate-resolution imaging spectroradiometer (MODIS) airborne simulator (MAS) that collects data in the visible and infrared bandwidths. The ER-2 also will be complemented by a P-3 aircraft that will carry a passive scanning radiometer (PSR) that, when used in conjunction with supplemental information about the land surface, will provide a detailed spatial map of shallow soil moisture. By functioning in this manner, the ER-2 and P-3 will serve as a proxy for a suite of measurements that in later years may be made routinely from satellite platforms. Detailed surface-based measurements of cloud structure also will be made in specific locations by radars at the ACRF Central Facility, a mobile 3-cm phased-array Doppler radar, and an array of 3-cm radars at fixed locations in the southern part of the domain.

\footnotetext{
${ }^{1}$ The SGP site is the largest permanent atmospheric research site in the world. Covering 55,000 square miles in Oklahoma and Kansas, it includes a highly instrumented Central Facility supplemented by smaller instrument clusters at 23 extended facilities. The site is managed by the U.S. Department of Energy's Atmospheric Radiation Measurement (ARM) Program. See http://www.arm.gov/sites/sgp.stm
} 
The second thrust of the experiment strategy involves many additional instrumented aircraft that will be used to sample regional-to-local scale processes. These aircraft will be deployed in a coordinated fashion to sample regions of interest and, for short periods, regions beneath the flight trajectory of the faster-moving ER-2. These aircraft will make a variety of measurements ranging from detailed assays of cloud microphysics and aerosols to high-resolution surveys of soil moisture and carbon fluxes.

The final thrust of CLASIC will be to provide a detailed characterization of the spatial and temporal evolution of the surface energy balance, soil moisture, carbon fluxes, and vegetation characteristics over the ACRF SGP domain using a network of four heavily instrumented surface stations (supersites). Each supersite will make multiple measurements at specific locations within a region of a few kilometers radius (intrasite measurements) that will be designed to characterize microscale and mesoscale variability. The evolution of atmospheric boundary layer flux profiles over these supersites and in other locations will be measured with a specially instrumented helicopter. These helicopter measurements will be used to relate the intrasite measurements at each supersite and to understand the evolution of the flux profiles. The links between these flux profiles and the accompanying cloud structure will be explored using measurements from fixed-wing aircraft. The helicopter also will measure the flux structure in other regions of opportunity across the ACRF SGP domain during CLASIC.

Data from these supersites and other regions that are sampled during the campaign will be combined with ER-2 and P-3 measurements, satellite data, and land surface modeling to produce spatial patterns of land-atmosphere fluxes that can be compared with the spatial cloudiness patterns. The measurement approach adopted during CLASIC will produce a rich data set that can be used to statistically analyze feedbacks between the land surface and cloud structure over the ACRF SGP site at a range of applicable time and space scales that can be integrated to document these processes on a region-wide basis. The measurements made during CLASIC will be unique in that they will crosscut many disciplines within the field of climate science. In this respect, CLASIC is one of the first efforts to simultaneously characterize the atmospheric boundary layer, land surface, and biosphere in an effort to better understand the links that exist between them during the early stages of cumulus convection. 


\section{CLASIC Science Questions}

There are five specific science questions that will be addressed during CLASIC:

\section{CLASIC Science Questions}

(1) What are the roles of cumulus convection and spatial variations in land cover in depleting low-level water vapor as it is advected into the SGP region?

(2) What are the relationships between cumulus clouds and the soil-plant-atmosphere exchange of heat, carbon, and water at the site?

(3) How do land cover changes, such as agricultural harvesting, impact surface heat, carbon, and water fluxes, and can those changes affect local and regional cumulus cloud formation at the ACRF SGP site?

(4) How do land surface processes at the SGP affect atmospheric aerosol loading and chemistry and what are the resulting effects on the microphysical and macrophysical properties of cumulus cloud fields?

(5) How well do the long-term (15+ years) surface flux measurements made at specific locations within the ACRF SGP represent the actual distribution of the fluxes across the domain?

\subsection{Science Question 1-What are the roles of cumulus convection and spatial variations in land cover in depleting low-level water vapor as it is advected into the SGP region?}

\subsubsection{State of the Science}

A climatologically significant atmospheric moisture gradient is observed across the ACRF SGP site (Rasmusson 1968; 1971). This moisture gradient, which has increasing values from southwest to northeast, is related to the geographic proximity of the Gulf of Mexico moisture source, which lies to the southeast, and the Rocky Mountains moisture barrier, which lies to the west. It is among the most significant atmospheric moisture gradients on Earth.

Globally, cloud formation is linked closely to the partitioning of the surface fluxes and soil moisture, and clouds have a propensity to initially form in regions where large sensible heat fluxes coincide with adequate moisture. For example, fair-weather clouds are enhanced over deforested patches in the Amazon basin where the sensible heat flux is greater (Chagnon et al. 2004). Other studies have shown that the height of the lifting condensation level and the time of cumulus initiation are related to boundary layer structure, which is determined by the distribution of the fluxes (Peppler and Lamb 1989; Berg and Kassianov 2007). Therefore, it is reasonable to postulate that the exact location of the SGP climatological moisture gradient during a given season may depend on many factors, including cloud and land surface interactions.

Spring and summer convection often is observed to the east of the SGP moisture gradient, less frequently to the west. The summer months at the ACRF SGP site are characterized by 
intermittent flow from the southeast. This flow configuration transports moisture from the Gulf of Mexico to the site, thereby increasing the specific humidity and relative humidity in the lower levels of the atmosphere. Since relative humidity and the associated lifting condensation level of warm parcels of air leaving the surface are important determinants of the timing and evolution of cumulus convection, flow from the southeast has a tendency to produce more clouds and precipitation, particularly over the southeast quadrant of the ACRF SGP site, but also at the ACRF SGP Central Facility.

The amount of moisture transported from the Gulf of Mexico to the ACRF SGP site depends largely on the nature of the atmospheric boundary layer development along the trajectory from the Gulf of Mexico. Different types of landscape variability, such as topography, land surface moisture, albedo, and vegetation, likely can significantly affect boundary layer and cloud structure along this trajectory by altering the partitioning of the turbulent sensible and latent heat fluxes, and their vertical profiles. Also, moisture may be added to the boundary layer through the evaporation of surface water and plant transpiration of soil moisture, further altering the state of the boundary layer. Along the trajectory from the Gulf of Mexico, cumulus clouds that form in the air mass redistribute moisture by transporting it from the boundary layer to the free atmosphere. At the same time, entrainment at the boundary layer top introduces drier air into the boundary layer from the free atmosphere above. If cumulus clouds develop in the air mass en route from the Gulf of Mexico, precipitation may result and thus remove moisture from the air mass to increase the standing water on the surface and the soil moisture. Because the amount of boundary layer moisture is a key regulator of cloud formation and a key mechanism through which cloud and land surface feedbacks may manifest, the details of boundary layer and cloud development in air masses that are advected from the Gulf of Mexico have a significant impact on cloud conditions and radiative forcing at the ACRF SGP site.

Experience within the ARM Program suggests models have difficultly accurately representing the moisture transport and occurrence of convective clouds over the ACRF SGP site. Recent studies have shown that models tend to under predict the low-level moisture at the ACRF SGP Central Facility, thereby leading to errors in the predicted cloud cover, radiative fluxes, and precipitation (Cheinet et al. 2005). These errors are related to air mass history, which suggests difficulty with the convective triggering or boundary layer structure in the models. Both of these model components are susceptible to improper characterization of land surface-atmosphere exchanges.

There are two key parts to this science question. The first part involves the Lagrangian evolution of the cloud, precipitation, and boundary layer structure of air masses moving from the Gulf of Mexico to the ACRF SGP site. The second part is a detailed understanding of the role of the land surface in shaping this evolution. Efforts will be made during CLASIC to observe this evolution, though not in a strictly Lagrangian framework. Calculations of back trajectories will be used to identify, in real-time, synoptic situations during which air is transported from the Gulf of Mexico to the ACRF SGP site. When these situations arise, aircraft will be used to sample the cloud, precipitation, and boundary layer structure along the trajectory. The detailed measurements made currently at the supersites, though they may not lie along the trajectory itself, will be used to help interpret the aircraft measurements. Further, process-level links between the surface supersites and the along-trajectory aircraft measurements may be accomplished by passing the aircraft over the supersites. It is recognized that the variety of 
conditions encountered along a given trajectory from the Gulf of Mexico will not be completely represented by conditions at the supersites. However, it is likely that enough information will be gained during CLASIC about the relations between aircraft measurements and process-level atmospheric structure to interpret the aircraft data along the trajectory from the Gulf of Mexico in the context of land surface structure.

\subsubsection{CLASIC Measurement Strategy}

The primary platform that will be used to address this science question is the ER-2 airplane. When back trajectory calculations suggest flow from the Gulf of Mexico to any of the three surface supersites, the ER-2 will fly along the trajectory and pass over the site or sites. It will collect a full range of information about the cloud structure using its cloud radar, lidar, and precipitation radar. This information, combined with data collected with the MAS, which is a solar and infrared spectrometer, will enable assays of aerosol and cloud microphysical structure. Data collected concurrently with MAS and with the PSR on the P-3 airplane will enable characterization of the soil moisture and spectral albedo of the underlying surface. Similarly, data from MAS and the cloud lidar will provide information about the boundary layer depth and integrated moisture along the trajectory. Thus, the ER-2 and P-3 data should provide an excellent snapshot of the evolution of the cloud and boundary layer structure along an atmospheric Lagrangian trajectory from the Gulf of Mexico, while also providing key information about the underlying land surface.

\subsection{Science Question 2-What are the relationships between cumulus clouds and the soil-plant-atmosphere exchange of heat, carbon, and water at the site?}

\subsubsection{State of the Science}

Land cover is known to partly control the fluxes of water and heat to the atmosphere. When the land cover includes vegetation, evapotranspiration may be a key component of these fluxes. Evapotranspiration is controlled by water availability, plant physiology, leaf stomata, and atmospheric state variables including incoming solar radiation, temperature, and humidity. As such, evapotranspiration may be strongly modulated by cloud coverage, lifetime, optical thickness, and precipitation efficiency. Specifically, the relationship between cumulus clouds and the ecosystem fluxes of $\mathrm{CO}_{2}$, water, and heat is known to be sensitive to the partitioning of solar radiation between direct and diffuse, and to the spectral distribution of the longwave radiative flux.

This sensitivity to the radiative partitioning and spectral distribution mostly is due to the varying efficiencies of photosynthesis across different plant varieties. The efficiency with which a plant conducts photosynthesis determines carbon uptake, so the response of an entire ecosystem to differing radiative fluxes is often expressed in terms of the Net Ecosystem Exchange (NEE) of $\mathrm{CO}_{2}$ (and $\mathrm{C}^{18} \mathrm{OO}$ ). It is known that clouds strongly influence the NEE, through their impacts on the solar radiation amount, its diffuse-to-direct ratio and photosynthetically active fraction, temperature, and vapor pressure deficit. More specifically, carbon uptake generally is larger under partly cloudy skies and cloudy skies in some ecosystems, due to enhanced leaf illumination, and the altered spectrum of incident solar radiation. Thus, clouds influence the NEE and the surface heat balance and interact with plant stomatal dynamics through which 
photosynthesis and transpiration are coupled. Changes in the surface energy balance and boundary layer moisture transport may, in turn, influence cloud processes.

Grasslands, which are more common than forests in the ACRF SGP region, show less of the above sensitivity to cloud cover according to recent studies (Still et al. 2005). The more muted response of grasslands to clouds is due to higher illumination rates over the body of the plant and relatively higher light use efficiency. Thus, it is expected that the surface fluxes of $\mathrm{CO}_{2}$, water, and heat will differ between grassland regions and forested regions. This is expected to result in differences in boundary layer development and cloud structure, all other things being equal.

Thus, it is critical for models to accurately represent cumulus convection in GCMs and regional climate models, due to the potential impacts on agricultural production and water availability. This situation requires that these models accurately represent the interactions between cumulus convection, radiation, the biosphere, and soil moisture. A main focus of CLASIC will be to understand how these interactive processes impact the local cloud and boundary layer structure.

\subsubsection{CLASIC Measurement Strategy}

Land surface flux and state measurements will be made across the dominant land covers during CLASIC. These measurements will be used, in conjunction with land use data, to estimate the spatial distribution of the integrated heat balance and the flux partitioning across the SGP domain. That information will be associated with detailed measurements of cloud structure to assess the impact on cloud/atmospheric dynamics. Critical factors that shape the partitioning of the sensible and latent heat fluxes in the atmospheric boundary layer will be monitored-canopy temperature, vapor pressure deficit, and soil moisture availability. Hyper-spectral and broadband spectral indices of vegetation will be measured to ensure accurate modeling of both energy and $\mathrm{CO}_{2}$ fluxes. These indices will include leaf area index, green and dry standing biomass, biomass residue, canopy height, and maximum light-use efficiency. Digital cameras also will be deployed at selected sites in order to monitor in near-real time the surface conditions as well as the cloud type and the onset of cloud formation. These observations will be especially useful for evaluating the effect of diffuse light on carbon uptake for various surface conditions. Distributed fluxes will be estimated through data assimilation and calculations with a land surface model.

Four supersites located at the ACRF SGP Central Facility, the Little Washita Watershed, Fort Cobb, and Okmulgee (ACRF Extended-Facility \#21) will be the focal point for the land surface measurements. The latter of these sites is located in a forested region and each of these super sites will contain several subsites distributed over distances of a few kilometers. Continuous measurements will be made at these sites for the duration of CLASIC, including measurements of soil moisture, rainfall, and upwelling and downwelling radiation. The multiple tower locations will help quantify flux variance within each land use zone and will provide statistics for examining flux differences across contrasting land use configurations. Intensive high-resolution measurements of soil moisture will be made for specific regions within the domain using in situ networks and new aircraft sensors. Application of soil moisture retrieval algorithms from PSR data sets and from satellite data will be used to characterize the regional distribution of soil moisture.

Measurements from these ground-based supersites will be complemented by a major aircraft campaign that will characterize the land surface state, atmospheric boundary layer, atmospheric 
chemistry (composition), and cloud structure. An instrumented helicopter will be used to measure the water, heat, and carbon fluxes over each of the supersites, on an alternating basis. It is anticipated that the helicopter will spend several days over each site, depending on conditions. Additional aircraft will collect in situ samples of cloud and aerosol properties, as well as concentrations of $\mathrm{CO}_{2}$ and other carbon cycle gases and isotopes over each supersite and across the SGP region. The ER-2 and P-3 aircraft will characterize the regional cloud and land surface structure on multiple days during CLASIC. Thus, virtually all of the platforms deployed during CLASIC will be used to address this science question.

\subsection{Science Question 3-How do land cover changes, such as agricultural harvesting, impact surface heat, carbon, and water fluxes, and can those changes affect local and regional cumulus cloud formation at the SGP site?}

\subsubsection{State of the Science}

Land cover type and land use management can have a strong influence on surface water and heat fluxes which, in turn, can influence air temperature and atmospheric boundary layer dynamics. In the SGP region, winter wheat accounts for about $20 \%$ of the land area (Cooley et al. 2005). The winter wheat harvest during mid-to-late June contributes to the most dynamic period of the year, when atmospheric and land surface conditions undergo rapid changes. It entails the conversion of large areas to relatively bare soil over very short time periods. The reduction in evapotranspiration and surface heat flux modifications are quite significant, but the impact on the boundary layer and cloud structure is largely unknown. The wheat harvest occurrence within CLASIC thus will offer an exciting opportunity to examine influences of land surface change on the thermodynamic structure of the atmospheric boundary layer, cloud formation, and aerosol interactions.

\subsubsection{CLASIC Measurement Strategy}

A combination of surface and aircraft measurements will be used to study the period immediately before, during, and after the harvest. The measurement strategy will be similar to that used for Science Question 2, except that efforts will be made to identify regions experiencing intense winter wheat harvesting that can be measured by the helicopter and multiple aircraft, including the ER-2 and P-3.

\subsection{Science Question 4-How do land surface processes at the SGP affect atmospheric aerosol loading and chemistry and what are the resulting effects on the microphysical and macrophysical properties of cumulus cloud fields?}

\subsubsection{State of the Science}

While it is generally believed that land surface moisture variability plays an important role in modulating the local development of shallow cumulus clouds (Rabin et al. 1990; Chen and Avissar 1994), it is also likely that variations in the aerosol loading and chemistry play a significant role in that cloud development. Aerosols may impact cumulus clouds by serving as cloud condensation nuclei (CCN). When the CCN number is high, more cloud droplets of smaller size may result from the same amount of cloud liquid water than when the number is lower. Higher numbers of smaller cloud droplets most likely reduce the conversion of cloud 
droplets to precipitation in cumulus clouds, thereby influencing their microphysical and macrophysical structure. Since it is likely that the number and composition of CCN are impacted by land surface conditions, it is possible that cloud structure and precipitation efficiency are affected through this pathway. There are many diverse sources of CCN associated with land use changes, including dust, soil particles, and anthropogenic aerosols from populated and industrialized areas. There may also be feedbacks between the terrestrial biosphere and the atmosphere that occur through biogenic pathways created by the emissions of biogenic aerosols and their precursors that become CCN.

The CCN activity spectrum quantifies the number of aerosol particles that may nucleate as a function of the supersaturation of the environment. Mostly, the updraft velocity determines the level of supersaturation. The nucleation rate of aerosols also is a function of their chemical properties and often is characterized by an "accommodation coefficient." Hence, it is reasonable to assume that local changes in the character of the land surface may produce changes in the aerosol activity spectrum that, in turn, influence cloud microphysical properties and development.

Cumulus clouds often are assumed, perhaps erroneously, to follow the same logical progression that leads to potential aerosol indirect effects in stratocumulus, despite their structure being somewhat different (Xue and Feingold 2006). Drizzle may not be as prevalent in cumulus clouds unless they are dissipating, so the dynamic feedbacks that accompany drizzle, including the potential for evaporating drizzle to stabilize the subcloud layer, may not operate in cumulus cloud fields. The radiative transfer characteristics of cumulus may be impacted more readily (in an adiabatic sense) than those of stratocumulus, due to higher cumulus updraft velocities that can nucleate smaller aerosol particles. Cumulus clouds also may be more susceptible to semiindirect effects, such as an alteration and stabilization of clear patches by absorbing aerosol such as black carbon and mineral dust (Hansen et al. 1997).

In the absence of clouds, there can be significant direct impacts of aerosols on ecosystem fluxes. It has been demonstrated that there is a strong response of the NEE to aerosol optical depth (Niyogi et al. 2004). When the aerosol optical depth increases, the amount of diffuse radiation increases while the total irradiance decreases. Increases in aerosol optical depth have been shown to be associated with increases in NEE in forested regions, but produce strong NEE decreases for grasslands. Hence, the response of the biosphere to aerosol loading is diverse and is expected to impact the development of the atmospheric boundary layer and cumulus clouds through the implied changes in the water and heat fluxes, as indicated by different rates of NEE.

Cloud and aerosol interactions are complicated by dynamic interactions between clouds and their environment. These interactions may include changes in the updraft structure and entrainment associated with forcing from the land surface, or involve modified synoptic scale forcing that results from the intertwining of cloud and aerosol interaction physics. Thus, there are several mechanisms through which the land surface may alter the microphysical and macrophysical structure of cumulus clouds.

\subsubsection{CLASIC Measurement Strategy}

The measurement strategy to be used to address this science question will involve a combination of surface-based in situ remote sensors and aircraft. Instruments at the SGP Central Facility will 
be used to dissect the microphysical structure of the early stages of cumulus convection over that supersite, and aircraft and surface measurements will be characterize the aerosols interacting with the clouds concerned. Past observational studies of cumulus convection at the SGP have been severely limited by the nature of the cloud microphysical data available (Kollias et al. 2005). The addition of a second cloud radar frequency and the implementation of advanced signal processing techniques for both cloud radars now enables cloud microphysical processes to be observed with a new level of detail at the Central Facility. Surface aerosol measurements made there also have been upgraded to include measurements of cloud droplet nucleation. One important strategy during CLASIC will be use of detailed aircraft aerosol measurements to assist in the interpretation of continuous measurements at the Central Facility. Of particular interest will be the relationships between surface aerosol measurements, aerosols measured near the base of cumulus clouds, and subsequent development of those clouds.

The relationships between aerosol composition and chemistry also will be intensively examined, especially as they relate to land surface characteristics. Aircraft operating beneath 20,000 feet will sample the local variability of cloud and aerosol structure, including key aspects of aerosol chemistry. Efforts will be made to link these measurements to underlying surface characteristics as measured from high altitude aircraft (approximately 60,000 feet) and satellite platforms, and quantified by models. It is expected that measurements made in the vicinity of the Central Facility will be a pivotal part of this component of CLASIC, but other measurements made near interesting land-surface-related aerosol gradients also will play an important role.

CLASIC also is collaborating with the U.S. Department of Energy's Atmospheric Sciences Program Cumulus Humilis Aerosol Processing Study (CHAPS), which will be conducted concurrently at SGP during June 2007. CHAPS will examine the impacts of Oklahoma City urbanization, which is another form of land surface change, on aerosol and cloud structures, distributions, and processes. CHAPS also will examine the processing of aerosols by cumulus clouds by attempting to relate aerosols in the subcloud layer to aerosols that have been detrained around the periphery of cumulus clouds. This work will involve assessments of the urban-torural transition. By having CLASIC and CHAPS co-located in Oklahoma, scientists from each campaign can use observations from the other to extrapolate their results to broader geographical areas.

Biogenic aerosol pathways will be examined by measuring the fraction of organic aerosols present in aircraft samples in conjunction with the amount of $\mathrm{CO}_{2}$. While this approach will have limitations, it may provide information that can be used to classify clouds by their potential biogenic aerosol forcing.

The primary platforms used during CLASIC to address this science question will be aircraft making detailed measurements of cloud and aerosol structure, along with instrumentation deployed permanently at the ACRF SGP Central Facility. Back trajectory calculations, aircraft aerosol measurements, and surface aerosol measurements will be the primary determinant of aerosol background state during CLASIC. 


\subsection{Science Question 5-How well do the long-term (+15 years) surface flux measurements made at specific locations within the ACRF SGP represent the actual distribution of the fluxes across the domain?}

\subsubsection{State of the Science}

A key element of the ARM research strategy is the use of single column models (SCMs) as a mechanism for testing GCM parameterizations. Use of an SCM involves, essentially, a climate model simulation for a single GCM grid-cell. Initialization of an SCM requires appropriate lateral and surface boundary conditions in the form of external, large-scale, forcing on the gridcell. It is well known that SCMs are quite sensitive to the specification of the underlying land surface forcing. The required SCM land surface forcing consists of the domain average latent, sensible, and radiative heat fluxes. These fluxes are quite variable across the SGP domain due to the wide diversity of land use and the climatological moisture gradient that divides the domain. Therefore, the ACRF SGP includes 23 distributed sites that make point measurements of the surface fluxes that are incorporated into an assimilation scheme (Variational Analysis) to produce the estimated land surface forcing on the grid-cell as a whole.

In addition to GCMs and SCMs, ARM also uses cloud system resolving models (CSRMs) to simulate the cloud structure over the SGP ACRF. Results from these models often are used to develop or test parameterizations that are eventually used in GCMs. Clouds are explicitly resolved in CSRMs on a scale of one kilometer or less, and some CSRMs contain sophisticated "bin" microphysical schemes that are able to simulate cloud and aerosol interactions. Some CSRMs also are coupled to complex land surface modules that include representations of vegetation, soil, and ground water. Land surface variations are known to modulate cloud structure in CSRM simulations and the results of these experiments are contributing to our knowledge of land surface-atmosphere interactions.

A typical approach used to characterize the land surface in CSRMs is to make point measurements of the fluxes over a diverse range of land use patterns, and then use assays of the land surface coverage over the domain to up-scale these point measurements and produce estimated spatial flux patterns across the domain. The current scheme used to characterize the land surface forcing over the ACRF SGP for ARM SCM applications follows this procedure quite closely. It uses weighted averages of point measurements of the fluxes at the 23 measurement locations, with the weighting factors determined by assays of the land-use patterns. Unfortunately, there are little previous observational data that can be used to evaluate these approaches, so a primary goal of CLASIC is to determine whether these up-scaling techniques are sufficiently accurate. A related goal is to determine if the current distribution of flux measurements is appropriate, given the distribution of land coverage.

\subsubsection{CLASIC Measurement Strategy}

Consistent with the above, the measurement strategy during CLASIC will be to use a helicopter to obtain detailed samples of the surface and boundary layer fluxes over a diverse range of land surface types, and to characterize the spatial distribution of soil moisture and surface albedo across the domain from aircraft and surface observations. These inputs, along with counterpart measurements from the fixed distributed sites, will be used in a sophisticated land surface model to estimate the variability of the fluxes on scales of less than $100 \mathrm{~m}$. Once the fluxes have been simulated for CLASIC using this model, domain averages will be computed and compared to 
those derived using the above standard ARM method of obtaining a weighted average from the 23 ACRF flux sites. The spatial flux distributions that result from this model then will be used as input for CSRM simulations for the CLASIC period. It is expected that the surface fluxes across the SGP ACRF will be sensitive to the harvesting pattern of winter wheat, so efforts will be made to make as many measurements as possible in regions where the harvest is particularly widespread.

To address this science question, the ER-2 and P-3 aircraft will be flown over the ACRF SGP site in a "survey" pattern as frequently as possible. This pattern will be a methodical trajectory that covers the entire domain and will be flown on mostly clear, partly cloudy, and cloudy days. This survey pattern will enable the evolution of the land surface cover, soil moisture, and albedo to be characterized across the SGP domain. Detailed surface flux measurements made at specific locations around the surface supersites will be complemented by spatial measurements of the near-surface fluxes and flux profiles collected with the helicopter. The helicopter will visit all of the surface supersites in addition to other locations of interest within the SGP domain. Efforts will be made to sample the spatial fluxes over as much of the SGP domain as is plausible within the operational and meteorological constraints. As noted above, a detailed land-surface model will serve as the integrator for all of the land surface measurements made during CLASIC.

\section{Concluding Remarks}

Interactions between the land surface, atmospheric boundary layer, and cumulus clouds represent a major unknown in the climate system. Our current knowledge of the processes that facilitate these interactions has been gained, largely, through the use of heavily parameterized models. Some processes that may produce changes in cumulus cloud structure, such as biogenic aerosol pathways, have been proposed only recently and their impact essentially is unknown. Through intensive measurements from surface, airplane, and satellite platforms, CLASIC will seek to quantify and characterize these interactions over the Southern Great Plains for June 2007. The measurement strategy of CLASIC is unique relative to past land surface-atmosphere interaction experiments, in that the combination of measurements is designed to facilitate a process-level understanding at a range of applicable space and time scales. It is designed to facilitate the interdisciplinary analyses needed to address some of the complex mechanisms through which the atmosphere and land surface communicate.

Each of the five science questions to be addressed by CLASIC involves a specific issue for which improved parameterizations of land surface-atmosphere interactions are needed in GCMs, SCMs, and CSRMs. The data set that will be collected during CLASIC will complement other international efforts by the ARM Program, such as in the Convective Orographic Precipitation Study and the African Monsoon Multidisciplinary Analysis Program, that are addressing interactions between the land surface, atmospheric boundary layer, and cumulus clouds in very different environments in other parts of the world. Thus, it is hoped that CLASIC and these companion experiments will reduce the current uncertainty of land surface forcing in GCMs. 


\section{References}

Berg, L. K., and E. Kassianov. 2007. Temporal variability of fair-weather cumulus statistics at the ACRF SGP site. J. Climate, (in revision).

Chagnon, FJF, RL Bras, and J Wang. 2004. "Climate shift of patterns of shallow cumulus over the Amazon.” Geophysical Research Letters, 31:L24212.

Cheinet, S, A Beljaars, M K h̆ler, J-J Morcrette, and P Viterbo. 2005. “Assessing the physical processes in the ECMWF model forecasts using the ARM SGP observations, ECMWF-ARM Report Series.” European Centre for Medium Range Forecasting, Shinfeld Park, Reading, England, p 29.

Chen, F, and R Avissar. 1994. "Impact of land-surface moisture variability on local shallow convective cumulus and precipitation in large-scale models.” Journal of Applied Meteorology, 33:1382-1401.

Cooley, HS, WJ Riley, MS Torn, and Y He. 2005. "Impact of agricultural practice on regional climate in a coupled land surface mesoscale model.” Journal of Geophysical ResearchAtmosphere, 110, D03113.

Hansen, J, M Sato, and R Reudy. 1997. “Radiative forcing and climate response.” Journal of Geophysical Research, 102(D6)6831-6864.

Kollias, P, EE Clothiaux, BA Albrecht, MA Miller, KP Moran, and KL Johnson. 2005. “The Atmospheric Radiation Measurement Program cloud profiling radars: An evaluation of signal processing and sampling strategies.” Journal of Atmospheric and Oceanic Technology, 22:930-940.

Niyogi, D, HI Chang, VK Saxena, T Holt, K Alapaty, F Booker, F Chen, KJ Davis, B Holben, T Matsui, T Meyers, WC Oechel, RA Pielke, R Wells, K Wilson, and YK Xue. 2004. "Direct observations of the effects of aerosol loading on net ecosystem $\mathrm{CO}_{2}$ exchanges over different landscapes.” Geophysical Research Letters, L20506.

Peppler, RA, and PJ Lamb. 1989. “On tropospheric static stability and central North American growing season rainfall.” Monthly Weather Review, 117:1156-1180.

Rabin, RM, S Stadler, PJ Wetzel, DJ Stensrud, and M Gregory. 1990. “Observed effects of landscape variability on convective clouds.” Bulletin of the American Meteorological Society, 71:272-280.

Rasmusson, EM. 1968. "Atmospheric water vapor transport and the water balance of North America. Part II: Large-scale water balance investigations.” Monthly Weather Review, 99:11-135. 
Rasmusson, EM. 1971. “A study of the hydrology of eastern North America using atmospheric vapor flux data.” Monthly Weather Review, 99:119-135.

Still, CJ, WJ Riley, SC Biraud, DC Noone, and JA Berry. 2005. “The impact of clouds on ecosystem-atmosphere $\mathrm{CO}^{18} \mathrm{O}$ exchanges in the U.S. Great Plains.” Eos, Transactions, American Geophysical Union, 86 Fall Meeting Assembly Supply, Abstract B22B-01.

Xue, H, and G Feingold. 2006. "Large-eddy simulations of trade wind cumuli: Investigation of aerosol indirect effects.” Journal of Atmospheric Science, 63:1605-1622. 